\title{
Effect of dietary fats rich in lauric, myristic, palmitic, oleic or linoleic acid on plasma, hepatic and biliary lipids in cholesterol-fed hamsters*
}

\author{
BY ELKE A. TRAUTWEIN, ANGELIKA KUNATH-RAU, JULIANE DIETRICH, \\ STEPHAN DRUSCH AND HELMUT F. ERBERSDOBLER
}

Institute of Human Nutrition and Food Science, University of Kiel, Düsternbrooker Weg 17, D-24105 Kiel, Germany

(Received 27 December 1995 - Revised 8 July 1996 - Accepted 13 July 1996)

\begin{abstract}
Effects of different dietary fats on plasma, hepatic and biliary lipids were determined in male golden Syrian hamsters (Mesocricetus auratus) fed on purified diets for 7 weeks. Diets were made by blending different fats containing characteristic fatty acids: butter (14:0 + 16:0), palm stearin (16:0), coconut oil (12:0 + 14:0), rapeseed oil (18:1), olive oil (18:1) and sunflowerseed oil (18:2). In all diets except the sunflowerseed oil diet dietary 18:2 was held constant at $2 \%$ energy. Total fat supplied 12 \% of energy and cholesterol was added at $4 \mathrm{~g} / \mathrm{kg}$ diet. Plasma cholesterol and triacyglycerol concentrations were increased by dietary cholesterol. After 7 weeks, plasma cholesterol concentrations were highest with the palm stearin, coconut oil and olive oil diets (8.9, 8.9 and $9.2 \mathrm{mmo} / \mathrm{h})$ and lowest with the rapeseed oil and sunflowerseed oil diets $(6.7$ and $5.5 \mathrm{mmo} / \mathrm{h})$ while the butter diet was intermediate (8.5 mmol/). Hepatic cholesterol concentration was highest in hamsters fed on the olive oil diet and lowest with the palm stearin diet (228 $v .144 \mu \mathrm{mol} / \mathrm{g}$ liver). Biliary lipids, lithogenic index and bile acid profile of the gall-bladder bile did not differ significantly among the six diets. Although the gallstone incidence was generally low in this study, three out of 10 hamsters fed on the palm stearin diet developed cholesterol gallstones. In contrast, no cholesterol gallstones were found with the other diets. Rapeseed and sunflowerseed oils caused the lowest plasma cholesterol and triacyglycerol concentrations whereas olive oil failed to demonstrate a cholesterol-lowering effect compared with diets rich in saturated fatty acids. Since 18:2 was kept constant at $2 \%$ of energy in all diets, the different responses to rapeseed and olive oils could possibly be attributed to their different contents of 16:0 (5.6 \% v. 12.8 \% respectively). Other possible explanations are discussed.
\end{abstract}

Plasma: Liver lipids: Dietary fat: Hamster

Syrian golden hamsters (Mesocricetus auratus) are commonly used to investigate lipid metabolism because they have a well-established similarity with human cholesterol and bile acid metabolism (Spady \& Dietschy, 1985; Spady et al. 1985). In addition, hamsters develop lithogenic bile and cholesterol gallstones in response to a variety of dietary manipulations. Generally, hamsters fed on purified diets with a modest level of fat (40-50 g/ $\mathrm{kg}$ ) and an excessive amount of cholesterol (3-4 g/ $\mathrm{kg}$ ) develop cholesterol gallstones, but the incidence is variable for genetic and dietary reasons (Dam, 1971; Cohen et al. 1989, 1992; Trautwein et al. 1993a). It is well established that excessive dietary cholesterol contributes to hyperlipidaemia, supersaturation of gall-bladder bile and cholesterol gallstone formation. However, the type of dietary fat and even specific fatty acids may also

* Part of this work was presented at the Experimental Biology 1995 meeting, Atlanta, Georgia, USA. 
influence cholelithiasis. The most commonly used model for gallstone induction in the hamster relied on butter as the source of fat. It was thought that butter was somehow essential for stone induction (Dam, 1971; Ayyad et al. 1992; Cohen et al. 1992; Trautwein et al. 1993a). However, substitution of olive oil, maize oil or safflowerseed oil for butterfat prevented cholesterol gallstones, whereas replacement of butter with palmitic acid further enhanced gallstone formation (Ayyad et al. 1992; Cohen et al. 1992). In contrast, substitution of 18:1-rich olive oil for saturated fatty acids (12:0, 14:0 and 16:0) in butterfat, palm stearin or coconut oil reduced cholesterol gallstone formation without affecting plasma lipids and the lithogenicity of the gall-bladder bile (Jonnalagadda et al. 1995).

The present study examined effects of dietary fats rich in saturated or unsaturated fatty acids on plasma, hepatic and biliary lipids using the cholesterol-fed hamster model. Diets were modified specifically to test whether substitution of monounsaturated (18:1) or polyunsaturated (18:2) fatty acids for saturated fatty acids (12:0, 14:0 and 16:0) would affect the metabolism of cholesterol and bile acids and cholesterol gallstone development. In all diets, except the diet rich in polyunsaturated fatty acids, dietary 18:2 was kept at $2 \%$ of energy to equalize and assure an adequate intake of the essential fatty acid. Because results from human studies suggest that differences may exist in the cholesterol-lowering effect of rapeseed oil and olive oil (Lichtenstein et al. 1993; Jones et al. 1994) these two sources of monounsaturated fatty acids were included in the present study.

\section{MATERIALS AND METHODS}

\section{Animals, diets and feeding procedures}

Male golden Syrian hamsters (Lakeview strain, Charles River, Sulzfeld, Germany) weighing approximately $50 \mathrm{~g}$ were randomly assigned to six diet groups $(n 10$ animals per diet group, except for the palm stearin diet with $n$ 11) and fed on purified diets containing $12 \%$ energy (en) as fat and $4 \mathrm{~g}$ dietary cholesterol $/ \mathrm{kg}$ for up to 7 weeks. The purified diets were made by blending different fats: butter (rich in 14:0+16:0), palm stearin (rich in 16:0), coconut oil (rich in 12:0 + 14:0), rapeseed oil (rich in 18:1 + 18:3), olive oil (rich in 18:1) and sunflowerseed oil (rich in 18:2). Dietary 18:2 was adjusted in all diets (except the sunflowerseed-oil and rapeseed-oil diets) to $2 \%$ en by adding safflowerseed oil. The fats and oils used for diet preparation were commercially available for human consumption (kindly provided by Unilever, Hamburg, Germany), except for rapeseed oil which was obtained as a non-refined oil from Raiffeisen HaGe, Kiel, Germany. The basal compositions of the purified diets are listed in Table 1 . The fatty acid profiles of the fat blends as analysed by GC are summarized in Table 2 .

The hamsters were housed in groups of three or four animals per cage and kept in a temperature-controlled environment with a $12 \mathrm{~h}$ light-dark cycle (lights on 18.00 hours). The diets were fed ad libitum ( $25 \mathrm{~g}$ wet weight/hamster per d) and the actual food consumption was recorded daily. Fresh water was provided daily ad libitum. Body weights were monitored on a weekly basis. All experimental protocols and procedures were approved by the Animal Care and Use Committee at the University of Kiel.

\section{Sample collection and analysis}

Dietary lipid absorption. Net lipid absorption was quantified by measuring the amount of dietary fat that was eaten, and the amount that was excreted in the faeces. The amount of food consumed was quantified on a daily basis throughout the experiment. For three 
Table 1. Composition ( $\mathrm{g} / \mathrm{kg}$ ) of the purified diets containing $4 \mathrm{~g}$ cholesterol/kg and different dietary fatty acids*

\begin{tabular}{|c|c|c|c|c|c|c|}
\hline $\begin{array}{l}\text { Fat ... } \\
\text { Characteristic fatty acids ... }\end{array}$ & $\begin{array}{c}\text { Butter } \\
14: 0+16: 0\end{array}$ & $\begin{array}{c}\text { Palm stearin } \\
16: 0\end{array}$ & $\begin{array}{l}\text { Coconut oil } \\
12: 0+14: 0\end{array}$ & $\begin{array}{c}\text { Rapeseed oil } \\
18: 1\end{array}$ & $\begin{array}{c}\text { Olive oil } \\
18: 1\end{array}$ & $\begin{array}{c}\text { Sunflower- } \\
\text { seed oil } \\
18: 2\end{array}$ \\
\hline Casein & 200 & 200 & 200 & 200 & 200 & 200 \\
\hline Maize starch & 385 & 385 & 385 & 385 & 385 & 385 \\
\hline Glucose & 200 & 200 & 200 & 200 & 200 & 200 \\
\hline Cellulose & 100 & 100 & 100 & 100 & 100 & 100 \\
\hline \multicolumn{7}{|l|}{ Fat } \\
\hline Safflowerseed oil $\dagger$ & 9 & 7 & 10 & - & 6 & - \\
\hline Butter & 41 & - & - & - & - & - \\
\hline Palm stearin & - & 43 & - & - & - & - \\
\hline Coconut oil & - & - & 40 & - & - & - \\
\hline Rapeseed oil & - & - & - & 50 & - & - \\
\hline Olive oil & - & - & - & - & 44 & - \\
\hline Sunflowerseed oil & - & - & - & - & - & 50 \\
\hline Mineral mix $\ddagger$ & 46 & 46 & 46 & 46 & 46 & 46 \\
\hline Vitamin mix $\S$ & 12 & 12 & 12 & 12 & 12 & 12 \\
\hline Cholinechloride & 3 & 3 & 3 & 3 & 3 & 3 \\
\hline Cholesterol & 4 & 4 & 4 & 4 & 4 & 4 \\
\hline
\end{tabular}

* Diets were fed as starch gels, prepared by withholding $60 \mathrm{~g} / \mathrm{kg}$ maize starch from the formulation and premixing it with $800 \mathrm{ml}$ simmering water to form a gel to which the remaining ingredients were added.

$\dagger$ To all diets (except the sunflowerseed and rapeseed oil diets) safflowerseed oil was added to equalize 18:2.

$\ddagger$ Ausman-Hayes mineral mix no. F8530, BioServ, Frenchtown, NJ, USA.

\$ Hayes-Cathcart vitamin mix. For details of the composition of the mix see Hayes et al. (1989).

Table 2. Fatty acid composition of the fat blends ( $\mathrm{g} / 100 \mathrm{~g}$ total fatty acids)*

(Values in parentheses represent percentage energy contributed by the individual fatty acid)

\begin{tabular}{|c|c|c|c|c|c|c|}
\hline Fatty acid & Butter & Palm stearin & Coconut oil & Rapeseed oil & Olive oil & $\begin{array}{c}\text { Sunflower- } \\
\text { seed oil }\end{array}$ \\
\hline $12: 0$ & $3.5(0.4)$ & $0.2(0.0)$ & $39.8(4.8)$ & $0.0(0.0)$ & $0.0(0.0)$ & $0.2(0.0)$ \\
\hline 14:0 & $11.2(1.3)$ & $1.1(0.1)$ & $15.0(1.8)$ & $0.0(0.0)$ & $0.0(0.0)$ & $0.2(0.0)$ \\
\hline $16: 0$ & $32.6(3.9)$ & $47.5(5.7)$ & $8.6(1.0)$ & $5.6(0.7)$ & $12.8(1.5)$ & $7.6(0.9)$ \\
\hline $16: 1$ & $1.8(0.2)$ & $0.3(0.0)$ & $0.0(0.0)$ & $0.0(0.0)$ & $1.1(0.1)$ & $0.0(0.0)$ \\
\hline 18:0 & $7.8(0.9)$ & $3.8(0.5)$ & $2.3(0.3)$ & $1.5(0.2)$ & $2.6(0.3)$ & $3.6(0.4)$ \\
\hline $18: 1$ & $19 \cdot 3(2.3)$ & $28.9(3.5)$ & $7.5(0.9)$ & $58.2(7.0)$ & $63.7(7.7)$ & $22.6(2.7)$ \\
\hline $18: 2$ & $16.2(2.0)$ & $16.8(2.0)$ & $17.2(2 \cdot 1)$ & $20.8(2.5)$ & $19.0(2.3)$ & $63.0(7.6)$ \\
\hline $18: 3$ & $0.7(0.1)$ & $0.2(0.0)$ & $0.1(0.0)$ & $11.4(1.4)$ & $0.1(0.0)$ & $1 \cdot 1(0.1)$ \\
\hline
\end{tabular}

* To all diets (except the sunflowerseed and rapeseed oil diets) safflowerseed oil was added to equalize 18:2.

consecutive days during week 4 faecal samples were collected from six randomly selected hamsters of each diet group which were housed individually in wire-bottomed cages. The total lipid contents of the diets and of the oven-dried faecal samples were determined gravimetrically. From these two values, the net absorption of dietary lipids was calculated as the difference between dietary intake and faecal excretion and expressed as a percentage of intake. 
Plasma lipid analysis. Blood samples were drawn from animals fasted overnight at baseline and after 3, 5 and 7 weeks. Blood was collected into EDTA-wetted syringes by cardiac puncture under light anaesthesia using a gaseous mixture of $\mathrm{CO}_{2}-\mathrm{O}_{2}(50: 50, \mathrm{v} / \mathrm{v})$, and plasma was separated immediately by centrifugation at $10000 \mathrm{~g}$ for 6 min. Plasma total cholesterol (TC), HDL-cholesterol (HDL-C) and triacylglycerol concentrations were determined by enzymic assays (Sigma kit no. 352 for cholesterol and no. 336 for triacylglycerol, Sigma Chemical, Deisenhofen, Germany). HDL-C was determined after precipitation of apo B- and apo E-containing lipoproteins with $\mathrm{Mg}^{2+}$-phosphotungstate using the HDL-cholesterol Reagent Set (Boehringer Diagnostica, Mannheim, Germany), according to the procedure of Weingard \& Daggy (1990).

Necropsy and gallstone evaluation. At 5 and 7 weeks, hamsters (five per diet group) were exsanguinated under anaesthesia, and the liver, caecum and the epididymal fat pad (right side only) were excised, blotted and weighed. A portion of the liver was frozen for hepatic cholesterol analysis. Gall-bladder bile was aspirated and analysed for biliary lipids and bile acids. The gall-bladder was dissected from the liver, opened under a dissecting microscope, and examined along with the remaining gall-bladder bile for cholesterol crystals, cholesterol and pigment gallstones under regular and polarized light by light microscopy as previously described (Hayes et al. 1989).

Hepatic cholesterol analysis. The hepatic cholesterol concentration was analysed in hamsters killed after 5 and 7 weeks following the procedure described previously (Trautwein et al. 1993b). TC was determined using a commercial assay (Sigma kit no. 352). Free cholesterol and cholesteryl esters were analysed by HPLC (Kim \& Chung, 1984). The esterified cholesterol concentrations were calculated as the difference between TC and free cholesterol.

Bile analysis. Gall-bladder bile lipids were isolated using a modified Folch extraction (Folch et al. 1957). A $10 \mu \mathrm{l}$ portion of gall-bladder bile was extracted with $3 \mathrm{ml}$ chloroform-methanol $(2: 1, \mathrm{v} / \mathrm{v})$ and $0.75 \mathrm{ml} 11.2 \mathrm{~g} / \mathrm{KCl}$ solution. Biliary cholesterol and phospholipids were determined with a commercially available assay in a portion of the chloroform phase using Wako Free Cholesterol C kit and Wako Phospholipid B kit (Wako Chemical, Düsseldorf, Germany). Biliary bile acids were analysed in a portion of the methanol-KCl phase as taurine- and glycine-conjugated bile acids using an isocratic HPLC method adapted from Rossi et al. (1987) as previously described (Trautwein et al. 1993b). Total bile acid concentration was calculated as the sum of individual bile acids (taurine and glycine conjugates of cholate, chenodeoxycholate and deoxycholate). The lithogenic index (LI) was calculated based on the relative molar ratios of lipid components and the actual total lipid concentration using a computer version of cholesterol solubility (Kuroki $e t$ al. 1986). The hydrophobicity index (HI) was calculated as the sum of the molar fraction of individual bile acids multiplied by their individual hydrophobicity index using the procedure of Heumann (1989).

Faecal bile acid and cholesterol excretion. Faecal samples were collected over a $3 \mathrm{~d}$ period from six randomly selected hamsters per diet group housed individually in wirebottomed cages to measure faecal bile acid and neutral sterol excretion. Faecal bile acids and neutral sterols were analysed in an oven-dried faecal sample using a modification of the method of Suckling et al. (1991) as described in detail previously (Trautwein et al. 1993b). Total bile acid concentration was determined enzymically using the Sigma bile acid kit no. 450 (Sigma). Neutral sterols were analysed by GC using a Fisons 9000 gas chromatograph equipped with a flame ionization detector and a capillary column (RTX$225,15 \mathrm{~m}, 0.25 \mathrm{~mm}$ ID, $0.25 \mu \mathrm{m}$ film thickness, Restek, Sulzbach, Germany). The column was operated at the following temperatures: $220^{\circ}$ for $25 \mathrm{~min}$ and $240^{\circ}$ for $15 \mathrm{~min}$. Injector 
temperature was set at $280^{\circ}$ and detector at $260^{\circ}$. He gas was used as the carrier at a flow rate of $1.3 \mathrm{ml} / \mathrm{min}$. A $1 \mu \mathrm{l}$ sample dissolved in $\mathrm{n}$-decane was injected using a split ratio of $30: 1$. Neutral-sterol standards including $5 \alpha$-cholestane, cholesterol, cholestanol, stigmasterol and stigmastanol were purchased from Sigma and coprostanol, coprostanone, cholestanone and $\beta$-sitosterol were obtained from Steraloids (Wilton, NH, USA). Neutral sterols were analysed as free sterols according to the method of Ausman et al. (1993).

Statistical analysis. Two-way ANOVA was used to determine diet, time and diet $\times$ time interaction effects for all measured variables at the two time points (e.g. plasma lipids, hepatic cholesterol, biliary lipids). One-way ANOVA was used to determine significant differences between diets within the 5 and 7 weeks data and for all the variables determined only at one time point (e.g. faecal bile acid and neutral sterol excretion). When significant $F$ ratios were found Scheffe's post-hoc test was used to determine differences between the diets. All statistical analyses were performed using the SuperANOVA statistical software package (Abacus Concepts Inc., Berkley, USA). Results were expressed as means and standard deviations. Differences were considered significant at $P<0 \cdot 05$.

\section{RESULTS}

\section{Food intake, growth, body and organ weights}

All hamsters were healthy throughout the feeding period and demonstrated normal weight gain. Ad libitum food consumption per hamster averaged $15.7 \mathrm{~g} / \mathrm{d}(8.7 \mathrm{~g}$ dry weight) with no apparent differences between diets. In addition, lipid absorption expressed as a percentage of intake was not significantly different between the diet groups (Table 3). No significant differences were noted in the initial and final body weight, the daily weight gain and the weight of the caecum and the epididymal fat pad (Table 3). Hamsters fed on the sunflowerseed oil diet gained less weight than those on the other diets but the difference was not statistically significant. Livers of hamsters fed on the butter and palm stearin diets were significantly heavier after 5 weeks compared with coconut and sunflowerseed-oil-fed hamsters but after 7 weeks no differences in liver weight existed between the six diets (Table 3).

\section{Plasma lipids}

Plasma cholesterol concentrations increased significantly with all diets compared with baseline, but to different extents (Table 4). After 3 weeks TC was significantly higher in hamsters fed on the palm stearin and butter diets compared with the diets rich in monounsaturated and polyunsaturated fatty acids (rapeseed oil, olive oil and sunflowerseed oil diets). Feeding the rapeseed oil diet resulted in the lowest cholesterol concentrations at 3,5 and 7 weeks. In contrast, hamsters fed on the olive oil diet showed a substantial increase in TC over the feeding period. After 7 weeks, plasma cholesterol concentrations were highest with the olive oil, palm stearin and coconut oil diets and lowest with the rapeseed oil and sunflowerseed oil diets while the butter diet was intermediate. The cholesterol concentration of the HDL fraction revealed, after 3 weeks only, significant differences between the diets. HDL-C tended to reflect the differences in the concentration of TC. At 5 and 7 weeks, HDL-C concentrations were no longer significantly different, although hamsters fed on the rapeseed oil and sunflowerseed oil diets had the lowest HDLC. The percentage HDL-C (HDL-C/TC) varied between 56 and $66 \%$ but did not differ substantially between the diets. 
Table 3. Food intake, lipid absorption, body-weight gain and organ weights of hamsters fed on purified diets containing $4 \mathrm{~g}$ cholesterol/kg and different dietary fatty acids*

(Mean values and standard deviations for five hamsters per diet and time point)

\begin{tabular}{|c|c|c|c|c|c|c|c|c|c|c|c|c|}
\hline & \multicolumn{2}{|c|}{$\begin{array}{c}\text { Butter } \\
14: 0+16: 0\end{array}$} & \multicolumn{2}{|c|}{$\begin{array}{c}\text { Palm stearin } \\
16: 0\end{array}$} & \multicolumn{2}{|c|}{$\begin{array}{l}\text { Coconut oil } \\
12: 0+14: 0\end{array}$} & \multicolumn{2}{|c|}{$\begin{array}{c}\text { Rapeseed oil } \\
\quad 18: 1\end{array}$} & \multicolumn{2}{|c|}{$\begin{array}{c}\text { Olive oil } \\
18: 1\end{array}$} & \multicolumn{2}{|c|}{$\begin{array}{l}\text { Sunflower- } \\
\text { seed oil } \\
18: 2\end{array}$} \\
\hline & Mean & SD & Mean & SD & Mean & SD & Mean & SD & Mean & SD & Mean & SD \\
\hline \multicolumn{13}{|l|}{ Food intake } \\
\hline Received (g/d) & 25 & & 25 & & 25 & & 25 & & 25 & & 25 & \\
\hline Consumed $(\mathrm{g} / \mathrm{d})$ & & & & & & & & & & & & \\
\hline & $16 \cdot 0$ & 1.6 & $15 \cdot 2$ & 1.6 & $15 \cdot 6$ & 1.4 & $15 \cdot 8$ & 1.8 & $16 \cdot 4$ & 1.6 & 15.1 & 1.5 \\
\hline during 7 weeks & $15 \cdot 6$ & 1.5 & $15 \cdot 2$ & 1.8 & 16.2 & 1.3 & 15.6 & $2 \cdot 1$ & $16 \cdot 5$ & 1.7 & 15.5 & 1.6 \\
\hline $\begin{array}{l}\text { Lipid absorption } \dagger \\
\text { (\% of intake) }\end{array}$ & 96 & 0.6 & 94 & 0.6 & 95 & 1.0 & 94 & 1.0 & 95 & 0.9 & 94 & 1.4 \\
\hline \multicolumn{13}{|l|}{ Body-weight gain } \\
\hline $\begin{array}{l}\text { Initial weight (g) } \\
5 \text { weeks }\end{array}$ & 51 & $2 \cdot 8$ & 51 & 3.3 & 51 & $5 \cdot 1$ & 52 & 3.9 & 52 & 4.5 & 52 & 3.5 \\
\hline Final weight $(\mathrm{g})$ & 132 & 9.8 & 130 & 14.6 & 119 & 6.8 & 132 & 9.5 & 128 & $7 \cdot 1$ & 112 & 12.3 \\
\hline Weight 8 & 2.3 & 0.3 & 2.2 & 0.5 & 1.8 & 0.1 & $2 \cdot 3$ & 0.2 & 2.2 & 0.3 & 1.7 & 0.4 \\
\hline \multicolumn{13}{|l|}{7 weeks } \\
\hline Final weight (g) $\ddagger$ & 127 & $16 \cdot 0$ & 125 & $10 \cdot 2$ & 135 & 9.7 & 128 & $7 \cdot 1$ & 137 & 17.0 & 126 & 9.8 \\
\hline Weight gain $(\mathrm{g} / \mathrm{d}) \S$ & 1.6 & 0.3 & 1.5 & 0.2 & 1.8 & 0.2 & 1.5 & 0.2 & 1.7 & 0.3 & 1.5 & 0.2 \\
\hline \multirow{2}{*}{\multicolumn{13}{|c|}{ Organ weights" }} \\
\hline $\begin{array}{l}5 \text { weeks } \\
\text { Liver (o) }\end{array}$ & $6.0^{\mathrm{a}}$ & 0.2 & $6.2^{\mathrm{ab}}$ & 0.9 & $5.0^{\mathrm{ab}}$ & & $5.6^{\mathrm{ab}}$ & 0.3 & 5. $2^{\mathrm{ab}}$ & & & \\
\hline & 1.9 & 0.2 & $\begin{array}{l}0.2 \\
1.9\end{array}$ & 0.3 & $\begin{array}{l}3.0 \\
1.7\end{array}$ & 0.4 & $\begin{array}{l}3.0 \\
1.8\end{array}$ & 0. & $\begin{array}{l}5 \cdot 2^{\mathrm{ab}} \\
1.7\end{array}$ & 0.4 & $4.9^{\mathrm{b}}$ & 0.8 \\
\hline Caecum (g) & 1.5 & 0.2 & 1.9 & 0.3 & 1.1 & 0.1 & 1.8 & & 1.7 & 0.2 & 1.5 & 0.2 \\
\hline Fat pad (g) & 1.5 & $0 \cdot 2$ & $1 \cdot 3$ & 0.3 & $1 \cdot 2$ & 0.1 & 1.6 & 0.3 & 1.4 & 0.3 & $1 \cdot 1$ & 0.4 \\
\hline \multicolumn{5}{|l|}{7 weeks } & & & & & & & & \\
\hline Caect & 1.6 & 0.1 & 1.8 & 0. & 1 . & 0. & 1. & 0. & 2.0 & 0.5 & 2.0 & 0.3 \\
\hline Fat pad $(\mathrm{g}) \mathrm{q}$ & 1.3 & 0.3 & 1.3 & 0.3 & 1.5 & 0.2 & 1.2 & 0.1 & 1.7 & 0.4 & 1.4 & 0.3 \\
\hline
\end{tabular}

a,b,c Mean values within a row not sharing a common superscript letter were significantly different, $P<0.05$ (one-way ANOVA and Scheffe's post-hoc test).

* For details of diets, see Tables 1 and 2.

$\dagger$ Lipid absorption was calculated as the difference between dietary fat intake and faecal fat excretion determined during week 4.

$\ddagger$ Two-way ANOVA revealed no diet, time, or diet $\times$ time interaction effects for final body weight.

$\S$ Two-way ANOVA showed no diet effect, but a time effect $(P<0.001)$ and a diet $\times$ time interaction $(P<0.023)$ for daily weight gain.

" Two-way ANOVA revealed no diet or time effect for caecal weight and weight of fat pad. For liver weight, no diet effect, but a time effect $(P<0.016)$ and a diet $\times$ time interaction $(P<0.019)$ were observed.

I Weight of the epididymal fat pad (right side only).

Plasma triacylglycerol concentrations also increased substantially compared with baseline, but because of the large variation within diet groups no significant differences could be observed between the diets, even though the rapeseed oil and sunflowerseed oil diets tended to decrease triacylglycerol concentrations at 7 weeks (Table 4).

\section{Hepatic cholesterol}

Both hepatic total and esterified cholesterol concentrations were increased with the cholesterol-rich diets compared with a normal cholesterol concentration of $8 \mu \mathrm{mol} / \mathrm{g}$ liver (E. A. Trautwein, unpublished results) and hepatic cholesterol accumulation was dependent on type of fat in the diet (Table 5). After 5 and 7 weeks the TC concentration in the liver 
Table 4. Plasma lipid concentrations (mmol/l) of hamsters fed on purified diets containing $4 \mathrm{~g}$ cholesterol/kg and different dietary fatty acids*

(Mean values and standard deviations for five hamsters per diet and time point)

\begin{tabular}{|c|c|c|c|c|c|c|c|c|c|c|c|c|}
\hline & \multicolumn{2}{|c|}{$\begin{array}{c}\text { Butter } \\
14: 0+16: 0\end{array}$} & \multicolumn{2}{|c|}{$\begin{array}{c}\text { Palm stearin } \\
16: 0\end{array}$} & \multicolumn{2}{|c|}{$\begin{array}{l}\text { Coconut oil } \\
12: 0+14: 0\end{array}$} & \multicolumn{2}{|c|}{$\begin{array}{c}\text { Rapeseed oil } \\
18: 1\end{array}$} & \multicolumn{2}{|c|}{$\begin{array}{c}\text { Olive oil } \\
18: 1\end{array}$} & \multicolumn{2}{|c|}{$\begin{array}{l}\text { Sunflower- } \\
\text { seed oil } \\
18: 2\end{array}$} \\
\hline & Mean & SD & Mean & SD & Mean & SD & Mean & SD & Mean & SD & Mean & SD \\
\hline \multicolumn{13}{|c|}{ Total cholesterol $\dagger$} \\
\hline Baseline & $2 \cdot 6$ & $0 \cdot 3$ & & & & & & & & & & \\
\hline 3 weeks & $9.3^{a}$ & 1.4 & $9.4^{\mathrm{ab}}$ & 2.5 & $7.7^{\mathrm{ab}}$ & 1.7 & $5.7^{b}$ & $1 \cdot 1$ & $6 \cdot 4^{\mathrm{ab}}$ & 1.4 & $6 \cdot 7^{\mathrm{ab}}$ & 0.6 \\
\hline 5 weeks & 7.4 & 1.8 & 9.4 & 2.6 & 6.4 & $1 \cdot 1$ & 5.8 & 1.2 & 7.0 & 0.8 & 6.4 & $1 \cdot 1$ \\
\hline 7 weeks & $8 \cdot 5$ & 0.7 & 8.9 & $1 \cdot 0$ & 8.9 & $1 \cdot 2$ & $6 \cdot 7$ & 0.2 & $9 \cdot 2$ & 1.8 & 7.5 & 1.2 \\
\hline \multicolumn{13}{|c|}{ HDL-cholesterol } \\
\hline 3 weeks & $7 \cdot 1^{\mathrm{a}}$ & 0.8 & $4.5^{b}$ & 0.9 & $5 \cdot 2^{\mathrm{ab}}$ & 1.6 & $3 \cdot 2^{b}$ & 1.0 & $5 \cdot 3^{\mathrm{ab}}$ & 0.6 & $4 \cdot 2^{b}$ & 0.6 \\
\hline 5 weeks & $3 \cdot 8$ & 1.9 & $5 \cdot 2$ & 1.4 & $4 \cdot 9$ & 0.8 & 3.4 & 1.6 & 4.6 & 0.8 & $3 \cdot 7$ & 0.4 \\
\hline 7 weeks & $5 \cdot 0$ & $1 \cdot 1$ & 5.9 & 0.7 & 5.6 & 1.6 & 4.4 & 1.2 & 5.6 & 0.7 & $4-2$ & 1.2 \\
\hline \multicolumn{13}{|c|}{ Triacylglycerols } \\
\hline Baseline & 1.8 & 0.4 & & & & & & & & & & \\
\hline 3 weeks & 6.4 & $3 \cdot 5$ & 3.7 & $2 \cdot 6$ & 3.1 & 1.7 & $2 \cdot 3$ & $1 \cdot 3$ & 2.9 & 1.0 & $2 \cdot 6$ & 0.6 \\
\hline 5 weeks & $4 \cdot 5$ & 1.7 & 3.4 & 1.4 & 2.0 & 0.5 & $2 \cdot 8$ & 0.8 & $2 \cdot 1$ & 0.4 & 2.6 & 1.1 \\
\hline 7 weeks & $2 \cdot 8$ & 0.8 & $2 \cdot 3$ & $1 \cdot 5$ & 3.0 & 1.2 & 1.6 & 0.2 & $4 \cdot 2$ & $2 \cdot 1$ & $2 \cdot 1$ & 1.4 \\
\hline
\end{tabular}

a,b,c Mean values within a row not sharing a common superscript letter were significantly different, $P<0$-05 (one-way ANOVA and Scheffe's post-hoc test).

* For details of diets, see Tables 1 and 2 .

$\lceil$ Two-way ANOVA revealed a diet effect $(P<0.001)$ and a time effect $(P<0.01)$ but no diet $x$ time interaction.

¥ Two-way ANOVA revealed a diet effect $(P<0.003)$ and a time effect $(P<0.024)$ but no diet $\times$ time interaction.

$\S$ Two-way ANOVA revealed a diet effect $(P<0.0031)$ but no time effect or diet $x$ time interaction.

was highest in hamsters fed on the olive oil and sunflowerseed oil diets, lowest with the palm stearin and coconut oil diets and intermediate in hamsters fed on the rapeseed oil and butter diets (Table 5). Most of the cholesterol that accumulated in the liver was in the form of esterified cholesterol which accounted for more than $90 \%$ of TC. Hepatic free cholesterol expressed as a percentage of TC was significantly higher in hamsters fed on the palm stearin diet compared with all other diets. Accordingly, esterified cholesterol concentration expressed as a percentage of TC was significantly lower with the palm stearin diet and accounted for only $91 \%$ of TC whereas for the other diets 93-95\% of TC was present in the form of esterified cholesterol (Table 5). The cholesteryl ester profile reflected partially the fatty acid profile of the dietary fats (Table 6). The palm stearin and coconut oil diets led to the highest percentage of cholesteryl palmitate (16:0), the rapeseed and olive oil diets were highest in cholesteryl oleate (18:1) which was the major cholesteryl ester in all diet groups. Livers from hamsters fed on the sunflowerseed oil diet showed the highest percentage of cholesteryl linoleate (18:2) which was increased $3 \cdot 1$-fold compared with the palm stearin diet and 1-7-fold compared with the coconut oil diet. Cholesteryl arachidonate (20:4) which accounted for less than $1 \%$ with the other diets was increased significantly to $1.8 \%$ with the rapeseed oil diet.

\section{Biliary lipids and bile acid profile}

After 5 weeks, no distinct differences were found in biliary lipids (both absolute concentrations of $\mathrm{mmol} / \mathrm{l}$ and as $\mathrm{mol} \%$ of bile acids, phospholipids and biliary cholesterol) or in the LI between the six diets (Table 7). The LI exceeded 1.0 for all diets but was higher 
Table 5. Hepatic cholesterol concentration ( $\mu$ mol/g liver) in hamsters fed on purified diets containing $4 \mathrm{~g}$ cholestero $/ \mathrm{kg}$ and different dietary fatty acids*

(Mean values and standard deviations for five hamsters per diet and time point)

\begin{tabular}{|c|c|c|c|c|c|c|c|c|c|c|c|c|}
\hline & \multicolumn{2}{|c|}{$\begin{array}{c}\text { Butter } \\
14: 0+16: 0\end{array}$} & \multicolumn{2}{|c|}{$\begin{array}{c}\text { Palm stearin } \\
16: 0\end{array}$} & \multicolumn{2}{|c|}{$\begin{array}{l}\text { Coconut oil } \\
12: 0+14: 0\end{array}$} & \multicolumn{2}{|c|}{$\begin{array}{c}\text { Rapeseed oil } \\
18: 1\end{array}$} & \multicolumn{2}{|c|}{$\begin{array}{c}\text { Olive oil } \\
18: 1\end{array}$} & \multicolumn{2}{|c|}{$\begin{array}{l}\text { Sunflower- } \\
\text { seed oil } \\
18: 2\end{array}$} \\
\hline & Mean & SD & Mean & SD & Mean & SD & Mean & SD & Mean & SD & Mean & SD \\
\hline \multicolumn{13}{|l|}{ After 5 weeks } \\
\hline Total cholesterol (TC) & $192^{\mathrm{ab}}$ & 22 & $151^{b}$ & 21 & $189^{\mathrm{ab}}$ & 16 & $193^{a b}$ & 37 & $222^{a}$ & 19 & $230^{\mathrm{a}}$ & 49 \\
\hline $\begin{array}{l}\text { Free cholesterol } \\
\text { \% of TC }\end{array}$ & \multicolumn{2}{|c|}{$6 \cdot 0^{\mathrm{b}}$} & $13^{\mathrm{a}}$ & $8^{a}$ & \multicolumn{2}{|c|}{$5.9^{\mathrm{b}}$} & \multicolumn{2}{|c|}{$5 \cdot 2^{b}$} & \multicolumn{2}{|c|}{$5.4^{\mathrm{b}}$} & \multicolumn{2}{|c|}{$5.4^{\mathrm{b}}$} \\
\hline $\begin{array}{l}\text { Esterified cholesterol } \dagger \\
\% \text { of TC }\end{array}$ & \multicolumn{2}{|c|}{$\begin{array}{c}180^{\mathrm{ab}}{ }_{94 \cdot 0^{\mathrm{b}}}^{21} \\
94\end{array}$} & $\begin{array}{l}137^{b} \\
91\end{array}$ & & \multicolumn{2}{|c|}{$\begin{array}{c}178^{\mathrm{ab}} 16 \\
94 \cdot 0^{\mathrm{b}}\end{array}$} & \multicolumn{2}{|c|}{$\begin{array}{c}183^{\mathrm{ab}}{ }^{37} \\
94 \cdot 8^{\mathrm{b}}\end{array}$} & \multicolumn{2}{|c|}{$\begin{array}{c}210^{\mathrm{a}} \quad 19 \\
94 \cdot 6^{\mathrm{b}}\end{array}$} & \multicolumn{2}{|c|}{$\begin{array}{c}218^{\mathrm{a}} \quad 49 \\
94 \cdot 6^{\mathrm{b}}\end{array}$} \\
\hline \multicolumn{13}{|l|}{ After 7 weeks } \\
\hline Total cholesterol & $203^{a b}$ & 21 & $144^{b}$ & 25 & $171^{\mathrm{ab}}$ & 25 & $195^{\mathrm{ab}}$ & 33 & $228^{\mathrm{a}}$ & 43 & $225^{2}$ & 22 \\
\hline Free cholesterol & $12^{a b}$ & & & 1.4 & $11^{a b}$ & 0.8 & & 0.7 & $13^{\mathbf{a b}}$ & $1 \cdot 1$ & $12^{\mathrm{ab}}$ & 1.0 \\
\hline $\begin{array}{l}\text { \% of TC } \\
\text { Esterified cholesterol } \dagger \\
\% \text { of TC }\end{array}$ & $191^{\mathrm{ab}}$ & 22 & $131^{b}$ & $\begin{array}{c}4^{\mathrm{a}} \\
24 \\
6^{\mathrm{a}}\end{array}$ & $\begin{array}{r}160^{\mathrm{ab}} \\
93\end{array}$ & $\begin{array}{c}7^{b} \\
24 \\
3^{b}\end{array}$ & $\begin{array}{r}184^{\mathrm{ab}} \\
94\end{array}$ & $4^{b 3}$ & $215^{\mathrm{a}}$ & & $213^{a^{5}}$ & $\begin{array}{l}6^{\mathrm{b}} \\
22 \\
4^{\mathrm{b}}\end{array}$ \\
\hline
\end{tabular}

a,b,c Mean values within a row not sharing a common superscript letter were significantly different, $P<0.05$ (one-way ANOVA followed by Scheffe's post-hoc test). Two-way ANOVA revealed only a diet effect $(P<0.0001)$ for total, free and esterified cholesterol, but no time effect and no diet $\times$ time interaction.

* For details of diets, see Tables 1 and 2.

$\uparrow$ Calculated as the difference between total and free cholesterol.

Table 6. Hepatic cholesteryl ester distribution (\%) of hamsters fed on purified diets containing $4 \mathrm{~g}$ cholesterol/kg and different dietary fatty acids*

(Mean values and standard deviations for five hamsters per diet and time point)

\begin{tabular}{|c|c|c|c|c|c|c|c|c|c|c|c|c|}
\hline & \multicolumn{2}{|c|}{$\begin{array}{c}\text { Butter } \\
14: 0+16: 0\end{array}$} & \multicolumn{2}{|c|}{$\begin{array}{c}\text { Palm stearin } \\
16: 0\end{array}$} & \multicolumn{2}{|c|}{$\begin{array}{c}\text { Coconut oil } \\
12: 0+14: 0\end{array}$} & \multicolumn{2}{|c|}{$\begin{array}{c}\text { Rapeseed oil } \\
18: 1\end{array}$} & \multicolumn{2}{|c|}{$\begin{array}{c}\text { Olive oil } \\
18: 1\end{array}$} & \multicolumn{2}{|c|}{$\begin{array}{c}\text { Sunflower- } \\
\text { seed oil } \\
18: 2\end{array}$} \\
\hline & Mean & SD & Mean & SD & Mean & SD & Mean & SD & Mean & SD & Mean & SD \\
\hline \multicolumn{13}{|c|}{ After 5 weeks } \\
\hline 20.4 & $0.2^{c}$ & 0.2 & $0.4^{b c}$ & 0.1 & $0.5^{\text {bc }}$ & 0.2 & $1 \cdot 7^{\mathrm{a}}$ & 0.1 & $0.5^{b c}$ & 0.2 & $0.7^{b}$ & 0.2 \\
\hline $18: 2$ & $10 \cdot 5^{b c}$ & 1.7 & $5 \cdot 7^{\mathrm{d}}$ & $1 \cdot 3$ & $11 \cdot 2^{\mathrm{ab}}$ & 1.3 & $7.4^{\text {cd }}$ & 1.0 & $6.9^{\mathrm{cd}}$ & 1.0 & $18 \cdot 4^{\mathrm{a}}$ & 2.7 \\
\hline $18: 1$ & $69.9^{b c}$ & $2 \cdot 1$ & $73 \cdot 5^{\mathrm{ab}}$ & $4 \cdot 3$ & $69 \cdot 6^{\mathrm{bc}}$ & $4 \cdot 1$ & $80 \cdot 1^{a}$ & 1.6 & $79.8^{a}$ & $1 \cdot 3$ & $65 \cdot 7^{\mathrm{c}}$ & $3 \cdot 5$ \\
\hline $18: 0$ & 7.6 & 4.4 & 4.0 & $3 \cdot 7$ & 4.8 & 2.9 & $4 \cdot 5$ & 0.9 & $5 \cdot 1$ & 0.5 & $5 \cdot 7$ & 0.8 \\
\hline $16: 0$ & $11 \cdot 8^{\mathrm{ab}}$ & $2 \cdot 4$ & $16 \cdot 4^{\mathrm{a}}$ & $3 \cdot 1$ & $13.9^{\mathrm{ab}}$ & 2.8 & $6 \cdot 2^{\mathrm{c}}$ & $1 \cdot 2$ & $7.7^{\mathfrak{e}}$ & 0.9 & $9 \cdot 4^{b c}$ & 0.7 \\
\hline \multicolumn{13}{|c|}{ After 7 weeks } \\
\hline $20: 4$ & $0.5^{b}$ & 0.1 & $0.4^{\mathrm{b}}$ & 0.1 & $0.5^{b}$ & 0.2 & $2 \cdot 0^{a}$ & 0.3 & $0 \cdot 4^{b}$ & 0.2 & $0.7^{\mathrm{b}}$ & 0.1 \\
\hline $18: 2$ & $8 \cdot 5^{b c}$ & 0.5 & $5 \cdot 9^{c}$ & 1.0 & $9.8^{b}$ & $1 \cdot 1$ & $7 \cdot 8^{\text {bc }}$ & 1.6 & $6 \cdot 7^{c}$ & $1 \cdot 1$ & $17.9^{\mathrm{a}}$ & 1.9 \\
\hline $18: 1$ & $71 \cdot 7^{\mathrm{abc}}$ & $2 \cdot 5$ & $69.7^{\mathrm{bc}}$ & 5.9 & $68.9^{c}$ & 4.6 & $79.4^{\mathrm{ab}}$ & $5 \cdot 1$ & $80 \cdot 4^{a}$ & 2.6 & $67.1^{c}$ & $3 \cdot 2$ \\
\hline $18: 0$ & 6.9 & $2 \cdot 2$ & $4 \cdot 5$ & 3.9 & $6 \cdot 3$ & 1.5 & $3-3$ & $2 \cdot 2$ & $4-5$ & $1 \cdot 1$ & 5.4 & 1.4 \\
\hline $16: 0$ & $12 \cdot 5^{b c}$ & 1.8 & $19 \cdot 4^{a}$ & $4 \cdot 1$ & $14 \cdot 4^{\mathrm{ab}}$ & 2.5 & $7.5^{c}$ & 1.4 & $7.9^{c}$ & $1 \cdot 1$ & $8.8^{c}$ & 0.6 \\
\hline
\end{tabular}

${ }^{a, b, c}$ Mean values within a row not sharing a common superscript letter were significantly different, $P<0.05$ (one-way ANOVA and Scheffe's post-hoc test). Two-way ANOVA revealed a diet effect $(P<0.0001)$ for all cholesterol esters, but no time effect or diet $\times$ time interaction.

* For details of diets, see Tables 1 and 2. 
Table 7. Biliary lipid concentration, lithogenic index of gall-bladder bile and gallstone incidence in hamsters fed on purified diets containing $4 \mathrm{~g}$ cholesterol/kg and different dietary fatty acids*

(Mean values and standard deviations for ten hamsters, except for the palm stearin group ( $n$ 11) and the rapeseed oil group $(n$ 9))

\begin{tabular}{|c|c|c|c|c|c|c|c|c|c|c|c|c|}
\hline & \multicolumn{2}{|c|}{$\begin{array}{c}\text { Butter } \\
14: 0+16: 0\end{array}$} & \multicolumn{2}{|c|}{$\begin{array}{c}\text { Palm stearin } \\
\text { 16:0 }\end{array}$} & \multicolumn{2}{|c|}{$\begin{array}{l}\text { Coconut oil } \\
12: 0+14: 0\end{array}$} & \multicolumn{2}{|c|}{$\begin{array}{l}\text { Rapeseed oil } \\
\qquad 18: 1\end{array}$} & \multicolumn{2}{|c|}{$\begin{array}{c}\text { Olive oil } \\
18: 1\end{array}$} & \multicolumn{2}{|c|}{$\begin{array}{c}\text { Sunflower- } \\
\text { seed oil } \\
18: 2\end{array}$} \\
\hline & Mean & SD & Mean & SD & Mean & SD & Mean & SD & Mean & SD & Mean & SD \\
\hline \multicolumn{13}{|l|}{ After 5 weeks } \\
\hline \multicolumn{13}{|l|}{ Biliary lipids $(\mathrm{mmol} / \mathrm{h})$} \\
\hline Bile acids $\dagger$ & 118 & 36 & 105 & 42 & 145 & 61 & 126 & 32 & 99 & 37 & 158 & 23 \\
\hline Phospholipids $\dagger$ & 13 & 1 & 12 & 3 & 14 & 5 & 17 & 6 & 13 & 5 & 21 & 3 \\
\hline Cholesterol $\uparrow$ & 8 & 1 & 7 & 2 & 9 & 3 & 7 & 2 & 6 & 3 & 9 & 0.4 \\
\hline \multicolumn{13}{|c|}{ Biliary lipids (mol \% of total biliary lipids) } \\
\hline Bile acids $\dagger$ & 85 & 3 & 84 & 4 & 86 & 3 & 84 & 3 & 84 & 2 & 84 & 2 \\
\hline Phospholipids $\dagger$ & 10 & 3 & 10 & 3 & 8 & 2 & 11 & 3 & 11 & 2 & 11 & 2 \\
\hline Cholesterol $\dagger$ & 6 & 1 & 6 & 1 & 6 & 2 & 5 & 1 & 5 & 1 & 5 & 1 \\
\hline Total lipids $(\mathrm{g} / \mathrm{l})^{\dagger}$ & 71 & 18 & 64 & 22 & 86 & 34 & 78 & 19 & 61 & 23 & 97 & 12 \\
\hline Lithogenic index $\dagger$ & 1.5 & 0.2 & 1.4 & 0.2 & 1.6 & 0.4 & $1 \cdot 1$ & 0.2 & $1 \cdot 2$ & 0.2 & 1.1 & 0.1 \\
\hline \multirow{2}{*}{\multicolumn{13}{|c|}{$\begin{array}{l}\text { After } 7 \text { weeks } \\
\text { Biliary lipids (mmol/l) }\end{array}$}} \\
\hline \multicolumn{4}{|l|}{ Biliary lipids (mmol/ $/)$} & & & & & & & & & \\
\hline Bile acids $\dagger$ & 169 & 53 & 188 & 17 & 156 & 39 & 109 & 25 & 131 & 34 & 176 & 46 \\
\hline Phospholipids $\dagger$ & $19^{\mathrm{ab}}$ & 6 & $22^{\mathrm{a}}$ & 3 & $19^{\mathrm{ab}}$ & 4 & $11^{b}$ & 3 & $18^{\mathrm{ab}}$ & 4 & $20^{\mathrm{ab}}$ & 4 \\
\hline Cholesterol $\dagger$ & $9^{\mathrm{ab}}$ & 3 & $11^{\mathrm{a}}$ & 1 & $9^{\mathrm{abc}}$ & 4 & $5^{\mathrm{c}}$ & 1 & $8^{\mathrm{abc}}$ & 2 & $9^{\mathrm{ab}}$ & 1 \\
\hline \multicolumn{13}{|c|}{ Biliary lipids (mol \% of total biliary lipids) } \\
\hline Bile acids $\dagger$ & $85^{\mathrm{ab}}$ & 2 & $85^{\mathrm{ab}}$ & 1 & $85^{\mathrm{ab}}$ & 1 & $87^{a}$ & 1 & $83^{b}$ & 1 & $85^{\mathrm{ab}}$ & 2 \\
\hline Phospholipids $\dagger$ & $10^{\mathrm{ab}}$ & 0.4 & $10^{\mathrm{ab}}$ & 1 & $10^{\mathrm{ab}}$ & 1 & $9^{\mathbf{a}}$ & 0.5 & $12^{\mathrm{b}}$ & 1 & $10^{\mathrm{ab}}$ & 1 \\
\hline Cholesterol $\dagger$ & 5 & 1 & 5 & $0-7$ & 5 & 0.5 & 4 & 0.6 & 5 & 0.8 & 5 & 1 \\
\hline Total lipids (g/l) & 101 & 31 & 114 & 10 & 94 & 23 & 64 & 14 & 81 & 20 & 106 & 26 \\
\hline Lithogenic index $\dagger$ & 1.2 & 0.3 & 1.2 & 0.2 & 1.2 & $0 \cdot 1$ & $1 \cdot 1$ & 0.2 & $1 \cdot 1$ & 0.2 & $1 \cdot 1$ & 0.3 \\
\hline \multicolumn{13}{|c|}{ Gallstone incidence (5 and 7 weeks combined) } \\
\hline Cholesterol stones/crystals & \multirow{2}{*}{\multicolumn{2}{|c|}{$0 / 10$}} & \multirow{2}{*}{\multicolumn{2}{|c|}{$3 / 11$}} & \multicolumn{2}{|c|}{$0 / 10$} & \multirow{2}{*}{\multicolumn{2}{|c|}{$0 / 9$}} & \multicolumn{2}{|c|}{$0 / 10$} & \multirow{2}{*}{\multicolumn{2}{|c|}{$0 / 10$}} \\
\hline Pigment stones & & & & & \multirow{2}{*}{\multicolumn{2}{|c|}{$\begin{array}{l}5 / 10 \\
5 / 10\end{array}$}} & & & \multirow{2}{*}{\multicolumn{2}{|c|}{$\begin{array}{l}3 / 10 \\
7 / 10\end{array}$}} & & \\
\hline No stones & \multicolumn{2}{|c|}{$\begin{array}{l}3 / 10 \\
7 / 10\end{array}$} & \multicolumn{2}{|c|}{$\begin{array}{l}2 / 11 \\
6 / 11\end{array}$} & & & \multicolumn{2}{|c|}{$8 / 9$} & & & \multicolumn{2}{|c|}{$5 / 10$} \\
\hline
\end{tabular}

a,b.c Mean values within a row not sharing a common superscript letter were significantly different, $P<0.05$ (one-way ANOVA and Scheffe's post-hoc test).

* For details of diets, see Tables 1 and 2.

$\dagger$ Two-way ANOVA revealed: for bile acids $(\mathrm{mmol} / 1)$ : a time effect $(P<0.007)$ but no diet effect and no diet $\times$ time interaction. For phospholipids (mmol/l) a diet effect $(P<0.037)$, a time effect $(P<0.005)$ and an interaction $(P<0.002)$. For biliary cholesterol (mmol/l): a diet effect $(P<0.0015)$, no time effect but an interaction $(P<0.014)$. For biliary lipids (bile acids, phospholipids and cholesterol) expressed as mol/100 mol no diet or time effect and no diet $\times$ time interaction was observed. For lithogenic index: a time effect $(P<0.011)$ but no diet effect and no diet $\times$ time interaction. For total lipids: a diet effect $(P<0.037)$, a time effect $(P<0.005)$ but no diet $\times$ time interaction.

with the coconut oil, butter and palm stearin diets. Neither the absolute concentration of total bile acids nor the relative distribution of individual bile acids (expressed as a percentage of total bile acid concentration) was remarkably different except for some minor variations between diets (Table 8). Also, no differences were observed in the HI.

\section{Gallstone incidence}

No cholesterol gallstones were observed with the butter, coconut oil, rapeseed oil, olive oil and sunflowerseed oil diets. By contrast, three of eleven hamsters fed on the palm stearin 
Table 8. Bile acid profile of gall-bladder bile from hamsters fed on purified diets containing $4 \mathrm{~g}$ cholesterol/kg and different dietary fatty acids*

(Mean values and standard deviations for five hamsters per diet and time point)

\begin{tabular}{|c|c|c|c|c|c|c|c|c|c|c|c|c|}
\hline & \multicolumn{2}{|c|}{$\begin{array}{c}\text { Butter } \\
14: 0+16: 0\end{array}$} & \multicolumn{2}{|c|}{$\begin{array}{c}\text { Palm stearin } \\
16: 0\end{array}$} & \multicolumn{2}{|c|}{$\begin{array}{l}\text { Coconut oil } \\
12: 0+14: 0\end{array}$} & \multicolumn{2}{|c|}{$\begin{array}{c}\text { Rapeseed oil } \\
18: 1\end{array}$} & \multicolumn{2}{|c|}{$\begin{array}{c}\text { Olive oil } \\
18: 1\end{array}$} & \multicolumn{2}{|c|}{$\begin{array}{c}\text { Sunflower- } \\
\text { seed oil } \\
18: 2\end{array}$} \\
\hline & Mean & SD & Mean & SD & Mean & SD & Mean & SD & Mean & SD & Mean & SD \\
\hline \multicolumn{13}{|l|}{ After 5 weeks } \\
\hline \multicolumn{13}{|l|}{$\%$ distribution } \\
\hline Taurocholate $f$ & 23.7 & 4.7 & 23.4 & 2.7 & $25 \cdot 0$ & $4 \cdot 1$ & $20 \cdot 2$ & 7.5 & 22.4 & $5 \cdot 3$ & $27 \cdot 6$ & 5.5 \\
\hline Glycocholate $\dagger$ & $16 \cdot 8^{b}$ & $3 \cdot 1$ & $17 \cdot 9^{\mathrm{ab}}$ & 1.6 & $19.7^{\mathrm{ab}}$ & $4 \cdot 1$ & $21.7^{a b}$ & 3.5 & $24 \cdot 5^{a}$ & 4.0 & $15 \cdot 3^{b}$ & 1.4 \\
\hline Taurocheno ${ }^{\dagger}$ & $26 \cdot 5^{a}$ & 5.6 & $23 \cdot 3^{\mathrm{ab}}$ & 2.0 & $22 \cdot 3^{\mathrm{ab}}$ & 6.0 & $18 \cdot 4^{\mathrm{ab}}$ & 3.9 & $15 \cdot 7^{b}$ & $4 \cdot 3$ & $26 \cdot 7^{a}$ & 2.2 \\
\hline Glycocheno $\dagger$ & $21 \cdot 5$ & $4 \cdot 5$ & 25.8 & 4.9 & $20 \cdot 0$ & $2 \cdot 6$ & $26 \cdot 5$ & 5.9 & $20 \cdot 0$ & $4 \cdot 3$ & 19.7 & $6 \cdot 1$ \\
\hline Taurodeoxycholate $†$ & 6.9 & $1 \cdot 0$ & 6.0 & 1.9 & 7.9 & 1.9 & $6 \cdot 3$ & 1.6 & $8 \cdot 3$ & $2 \cdot 3$ & $6 \cdot 1$ & $2 \cdot 0$ \\
\hline Glycodeoxycholate & $4 \cdot 5$ & 2.5 & $3 \cdot 6$ & 0.8 & 5.0 & $4 \cdot 0$ & 6.7 & 3.4 & $9 \cdot 0$ & $3 \cdot 2$ & $4 \cdot 7$ & $2 \cdot 4$ \\
\hline \multicolumn{13}{|l|}{ Ratios } \\
\hline Cholate:chenof & $0.9^{\mathbf{b}}$ & 0.1 & $0.8^{b}$ & $0 \cdot 1$ & $1 \cdot 1^{a b}$ & 0.1 & $0.9^{b}$ & 0.2 & $1 \cdot 3^{\mathrm{a}}$ & 0.1 & $0.9^{\mathrm{b}}$ & 0.2 \\
\hline Glycine:taurine ${ }^{\dagger}$ & $1 \cdot 2$ & 0.9 & 1.2 & 0.2 & 1.4 & $1 \cdot 0$ & $2 \cdot 4$ & 1.4 & $2 \cdot 0$ & 0.9 & 1.1 & 0.5 \\
\hline Primary:secondary $\dagger$ & 3.6 & 1.5 & $4 \cdot 4$ & $1 \cdot 3$ & 4.3 & 3.0 & $2 \cdot 4$ & 1.7 & 1.9 & 0.8 & $3 \cdot 3$ & $2 \cdot 0$ \\
\hline Hydrophobicity index $(\mathrm{HI}) \dagger$ & 0.31 & 0.02 & 0.31 & 0.02 & $0 \cdot 30$ & 0.02 & 0.32 & 0.03 & 0.30 & 0.02 & 0.30 & 0.03 \\
\hline \multicolumn{13}{|l|}{ After 7 weeks } \\
\hline \multicolumn{13}{|l|}{$\%$ distribution } \\
\hline Taurocholate $\dagger$ & 32.6 & 9.7 & $25 \cdot 0$ & 2.7 & 21.8 & $5 \cdot 3$ & $32-0$ & 1.0 & 25.9 & $6 \cdot 5$ & 31.2 & $2 \cdot 4$ \\
\hline Glycocholate ${ }^{\dagger}$ & $16 \cdot 6$ & 7.0 & 19.6 & 2.4 & 21.6 & $2 \cdot 2$ & $15 \cdot 7$ & 5.0 & $16 \cdot 2$ & 2.9 & 13.5 & $2 \cdot 4$ \\
\hline Taurocheno' & 27.3 & 6.4 & $25 \cdot 0$ & 2.8 & 20.4 & 4.5 & 27.9 & 5.6 & $25 \cdot 0$ & 5.4 & $30 \cdot 4$ & $3 \cdot 3$ \\
\hline Glycocheno† & $11 \cdot 1^{\mathrm{b}}$ & $6 \cdot 3$ & $19 \cdot 7^{\mathrm{a}}$ & 2.9 & $20 \cdot 9^{a}$ & 3.7 & $15 \cdot 4^{\mathrm{ab}}$ & 1.7 & $15 \cdot 7^{\mathrm{ab}}$ & $3 \cdot 3$ & $12 \cdot 2^{\mathrm{ab}}$ & $2 \cdot 3$ \\
\hline Taurodeoxycholate $f$ & $8 \cdot 2^{\mathrm{ab}}$ & $1 \cdot 2$ & $7 \cdot 3^{b}$ & 0.9 & $8 \cdot 1^{\mathrm{ab}}$ & 0.8 & $7 \cdot 3^{\mathrm{b}}$ & $1 \cdot 1$ & $10 \cdot 7^{\mathrm{a}}$ & $2 \cdot 2$ & $9 \cdot 2^{\mathrm{ab}}$ & 1.5 \\
\hline Glycodeoxycholate $†$ & $4 \cdot 2$ & $2 \cdot 8$ & 3.4 & $3 \cdot 1$ & $7 \cdot 2$ & $4 \cdot 4$ & 1.6 & 1.6 & $6 \cdot 5$ & $4 \cdot 3$ & 3.5 & 1.7 \\
\hline \multicolumn{13}{|l|}{ Ratios } \\
\hline Cholate:chenot & 1.3 & 0.2 & 1.0 & 0.1 & $1 \cdot 1$ & 0.1 & $1 \cdot 1$ & 0.2 & 1.0 & 0.1 & $1 \cdot 1$ & 0.1 \\
\hline Glycine:taurine $\dagger$ & 0.5 & 0.4 & 0.8 & 0.2 & $1 \cdot 1$ & 0.4 & 0.5 & 0.1 & 0.7 & 0.3 & 0.4 & $0 \cdot 1$ \\
\hline Primary:secondary $\dagger$ & 7.5 & $2 \cdot 0$ & $9 \cdot 1$ & 2.5 & 5.8 & $1 \cdot 7$ & $10 \cdot 4$ & 1.7 & $5 \cdot 5$ & $2 \cdot 5$ & $7 \cdot 1$ & 1.5 \\
\hline HI† & 0.27 & 0.03 & 0.29 & 0.02 & 0.31 & 0.03 & 0.27 & 0.02 & 0.31 & 0.03 & 0.29 & 0.01 \\
\hline
\end{tabular}

${ }^{\mathrm{ab}}$ Mean values within a row not sharing a common superscript letter were significantly different, $P<0.05$ (one-way ANOVA and Scheffe's post-hoc test).

* For details of diets, see Tables 1 and 2.

$\dagger$ Two-way ANOVA revealed: for taurocholate: a time effect $(P<0.035)$ but no diet effect and no diet $\times$ time interaction. For glycocholate: a diet effect $(P<0.005)$, a time effect $(P<0.033)$ and a diet $\times$ time interaction $(P<0.012)$. For taurocheno: a diet effect $(P<0.002)$, a time effect $(P<0.003)$ but no diet $\times$ time interaction. For glycocheno: a diet effect $(P<0.004)$, a time effect $(P<0.0001)$ but no diet $\times$ time interaction. For taurodeoxycholate: a diet effect $(P<0.003)$, a time effect $(P<0.0007)$ but no diet $\times$ time interaction. For glycodeoxycholate: a diet effect $(P<0.030)$ but no time effect and no diet $\times$ time interaction. For the cholate:cheno ratio: a diet effect $(P<0.008)$, a time effect $(P<0.013)$ and a diet $\times$ time interaction $(P<0.0001)$. For the glycine:taurine ratio: a diet effect $(P<0.04)$, a time effect $(P<0.0005)$ and a diet $\times$ time interaction $(P<0.025)$. For the primary:secondary bile acids ratio: a diet effect $(P<0.0044)$ but no time effect and no diet $\times$ time interaction. For HI: no diet effect, but a time effect $(P<0.012)$ and a diet $\times$ time interaction $(P<0.014)$.

diet showed cholesterol crystals when examined under polarized light microscopy (Table 7). Pigment stones (in a variety from greenish gel to well-formed green-brown to black stones) were found in hamsters from all diet groups; hamsters fed on the coconut oil and sunflowerseed oil diets showed the highest incidence $(50 \%)$ while only in one of nine hamsters fed on the rapeseed oil diet were pigment stones present.

\section{Faecal output and bile acid and neutral sterol excretion}

Faecal 3 d output (weight) and moisture content were similar among the diet groups and ranged from $0.93 \mathrm{~g} / \mathrm{d}$ with butter to $1.2 \mathrm{~g} / \mathrm{d}$ with olive oil. Hamsters fed on the rapeseed oil 
Table 9. Faecal bile acid and neutral sterol excretion in hamsters fed on purified diets containing $4 \mathrm{~g}$ cholesterol/kg and different dietary fatty acids*

(Mean values and standard deviations for six hamsters per diet. Hamsters were randomly selected and housed individually in wire-bottomed cages for $3 \mathrm{~d}$ during faecal collection)

\begin{tabular}{|c|c|c|c|c|c|c|c|c|c|c|c|c|}
\hline & \multicolumn{2}{|c|}{$\begin{array}{c}\text { Butter } \\
14: 0+16: 0\end{array}$} & \multicolumn{2}{|c|}{$\begin{array}{c}\text { Palm stearin } \\
16: 0\end{array}$} & \multicolumn{2}{|c|}{$\begin{array}{l}\text { Coconut oil } \\
12: 0+14: 0\end{array}$} & \multicolumn{2}{|c|}{$\begin{array}{c}\text { Rapeseed oil } \\
18: 1\end{array}$} & \multicolumn{2}{|c|}{$\begin{array}{c}\text { Olive oil } \\
18: 1\end{array}$} & \multicolumn{2}{|c|}{$\begin{array}{l}\text { Sunflower- } \\
\text { seed oil } \\
18: 2\end{array}$} \\
\hline & Mean & SD & Mean & SD & Mean & SD & Mean & SD & Mean & SD & Mean & SD \\
\hline \multicolumn{13}{|l|}{ Faecal excretion } \\
\hline $\begin{array}{c}\mathrm{g} / 3 \mathrm{~d}(\text { dry wt) } \\
\text { Neutral sterol exc }\end{array}$ & 2.78 & 0.44 & 2.99 & 0.71 & 3.28 & 0.41 & 3.50 & 0.64 & 3.57 & 0.64 & 2.93 & 0.30 \\
\hline$\mu \mathrm{mol} / \mathrm{g}$ faeces & 44.4 & 5.4 & 41.6 & 4.0 & 44.0 & 3.7 & 45.6 & 3.2 & 39.7 & $4 \cdot 1$ & 37.7 & 3.5 \\
\hline$\mu \mathrm{mol} / \mathrm{d}$ & $41 \cdot 5^{\mathrm{ab}}$ & $10 \cdot 3$ & $41 \cdot 0^{\mathrm{ab}}$ & 7.2 & $48 \cdot 1^{\mathrm{ab}}$ & 7.4 & $53 \cdot 0^{\mathrm{a}}$ & $8 \cdot 2$ & $47.0^{\mathrm{ab}}$ & 7.3 & $36 \cdot 7^{b}$ & 7.4 \\
\hline \multicolumn{13}{|c|}{ Distribution of individual neutral sterols ( $\mu \mathrm{mol} / \mathrm{g}$ faeces) } \\
\hline Cholesterol & $35 \cdot 2^{\mathrm{ab}}$ & $4 \cdot 1$ & $30 \cdot 6^{\mathrm{ab}}$ & 2.4 & $33 \cdot 1^{\mathrm{ab}}$ & $2 \cdot 6$ & $36 \cdot 1^{\mathrm{a}}$ & $2 \cdot 4$ & $34 \cdot 3^{\mathrm{ab}}$ & $4 \cdot 2$ & $29 \cdot 0^{\mathrm{b}}$ & $2 \cdot 6$ \\
\hline Coprostanol & $8 \cdot 5^{\mathrm{ab}}$ & 1.9 & $10 \cdot 2^{\mathrm{a}}$ & 1.9 & $9.8^{\mathrm{a}}$ & 1.4 & $6 \cdot 5^{\mathrm{ab}}$ & 1.7 & $4 \cdot 2^{b}$ & 4.5 & $7.0^{\mathrm{ab}}$ & 1.2 \\
\hline Cholestanol & 0.48 & 0.25 & 0.42 & 0.33 & 0.43 & 0.22 & 0.45 & 0.06 & 0.23 & 0.27 & 0.33 & 0.26 \\
\hline Cholestanone & $0.32^{c}$ & 0.29 & $0.32^{c}$ & 0.28 & $0.68^{b c}$ & 0.22 & $2.05^{a}$ & 0.40 & $0.47^{\mathrm{c}}$ & 0.40 & $1 \cdot 34^{\mathrm{b}}$ & 0.43 \\
\hline$\beta$-Sitosterol & ND & & ND & & ND & & 0.57 & 0.41 & 0.40 & 0.35 & $\mathrm{ND}$ & \\
\hline
\end{tabular}

ND, not detectable.

a,b,c Mean values within a row not sharing a common superscript letter were significantly different, $P<0.05$ (one-way ANOVA and Scheffe's post-hoc test).

* For details of diets, see Tables 1 and 2.

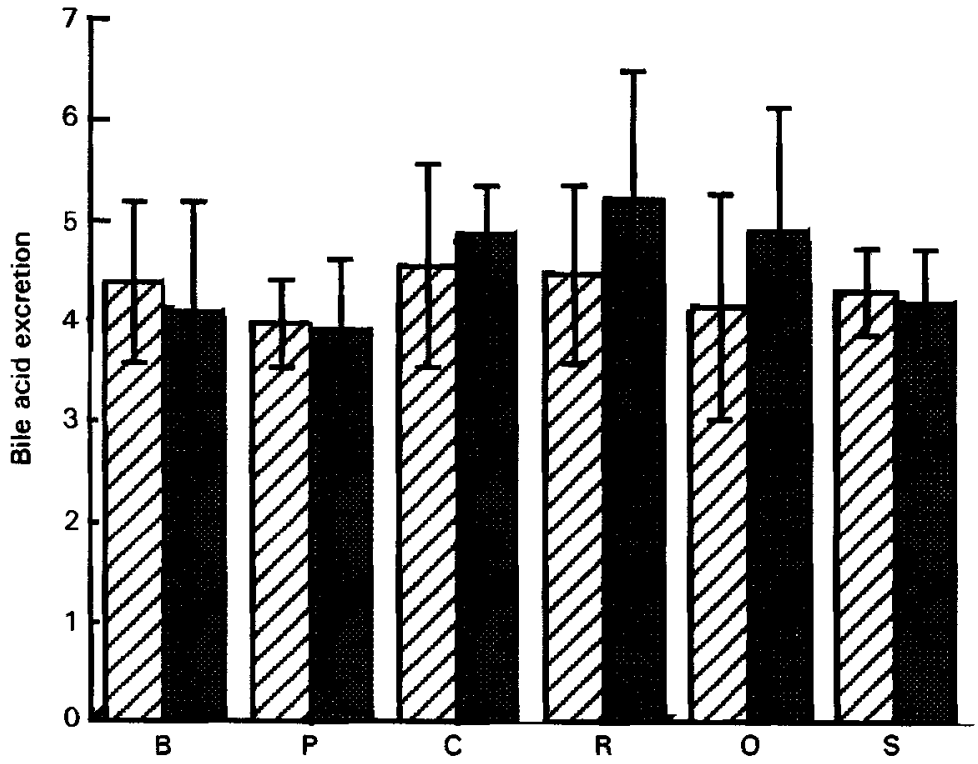

Fig. 1. Faecal total bile acid excretion expressed as $\mu \mathrm{mol} / \mathrm{g}$ faeces $(\mathbb{Z})$ and $\mu \mathrm{mol} / \mathrm{d}$ (圈) from hamsters fed on purified diets containing $4 \mathrm{~g}$ cholesterol/ $\mathrm{kg}$ and different dietary fatty acids. Fats studied were butter (B); palm stearin (P); coconut oil (C); rapeseed oil (R); olive oil (O); sunflowerseed oil (S). Values are means for six randomly selected hamsters per diet group housed individually during the $3 \mathrm{~d}$ faecal collection in wire-bottomed cages. Standard deviations are represented by vertical bars. No significant differences were found between diets as determined by oneway ANOVA. 
and olive oil diets tended to have a greater, but not significantly higher, faecal output (Table 9). Faecal total bile acid concentrations expressed as $\mu \mathrm{mol} / \mathrm{g}$ faeces or as $\mu \mathrm{mol} / \mathrm{d}$ did not differ significantly among diet groups (Fig. 1). However, faecal daily bile acid excretion was lowest in hamsters fed on the palm stearin and butter diets whereas the rapeseed oil diet resulted in the highest bile acid excretion on a daily basis $(5 \cdot 21 \mu \mathrm{mol} / \mathrm{d})$. Faecal total neutral sterol excretion expressed per $\mathbf{g}$ faeces was highest in hamsters fed on the rapeseed oil diet whereas sunflowerseed oil caused the lowest excretion of neutral sterols (Table 9). When expressed on a daily basis total neutral sterol excretion was again highest in hamsters fed on rapeseed oil and lowest in hamsters fed on sunflowerseed oil. Notably, the distribution of individual neutral sterols excreted in the faeces differed markedly between the six diet groups. Because of the excessive dietary cholesterol load, cholesterol was the major neutral sterol excreted and accounted for more than $70 \%$ of the total neutral sterols. Coprostanol, the main breakdown product of cholesterol, was the second major neutral sterol and was moderately higher in faeces from animals fed on the saturated fats especially palm stearin and lower in hamsters fed on the diets rich in monoand polyunsaturated fatty acids. Interestingly, cholestanone, which was only a minor sterol (1-2 \% of total sterols) excreted in faeces from hamsters fed on the butter, palm stearin, coconut and olive oil diets, was significantly higher with rapeseed and sunflowerseed oil (Table 9). In addition, $\beta$-sitosterol was only found in faecal samples from hamsters fed on rapeseed and olive oil and also in traces in animals fed on the sunflowerseed oil diet whereas this plant sterol was not found with the diets rich in saturated fatty acids.

\section{DISCUSSION}

We have investigated the impact of mono (MUFA)- $v$. polyunsaturated fatty acid (PUFA)rich fats in comparison with saturated fats on cholesterol and bile acid metabolism and their possible impact on cholesterol gallstone formation in a hamster model. Although the incidence of cholesterol gallstone formation was less than expected, nevertheless the results revealed important differences in plasma and hepatic lipids, especially between the two dietary MUFA-rich fats, rapeseed oil and olive oil.

The substantial increase in plasma cholesterol seen with all diets compared with baseline values was mainly the result of the excessive dietary cholesterol intake. The $4 \mathrm{~g}$ dietary cholesterol $/ \mathrm{kg}$ is equivalent to about five times the endogenous cholesterol synthesis rate (Spady et al. 1993) and will, therefore, overwhelm regulatory mechanisms and so result in a massive increase in plasma cholesterol, especially LDL-cholesterol. However, the extent of the increase in plasma cholesterol was affected by the type of dietary fat, suggesting that there is a dietary fat-cholesterol interaction. Feeding sunflowerseed oil (PUFA) and rapeseed oil (MUFA) resulted in plasma cholesterol concentrations below those for diets rich in saturated fatty acids (palm stearin, coconut oil, butter). Unexpectedly, olive oil failed to lower cholesterol, contrary to previous observations in hamsters (Jones et al. 1990; Sessions \& Salter, 1994). The cholesterolrich diets also induced a marked increase in the plasma triacyglycerol concentration. Specifically, the diets rich in saturated fats and the olive oil diet produced hypertriacylglycerolaemia after 7 weeks, a condition often observed in the cholesterolfed hamster (Hayes et al. 1992; Trautwein et al. 1993a). In contrast, feeding rapeseed and sunflowerseed oils did not elevate, or only minimally increased, triacylglycerol concentrations compared with baseline levels, and so attenuated the hypertriacylglycerolaemic response to cholesterol. 
It is well accepted that in the hamster model dietary cholesterol results in a marked increase in the LDL-cholesterol concentration because of a down-regulation of the LDL receptor (Dietschy et al. 1993; Spady et al. 1993). Saturated fatty acids further augment the hypercholesterolaemic effect, whereas unsaturated fatty acids normally attenuate the effect by partially restoring the suppressed hepatic LDL-receptor activity. However, it is also known from numerous studies using different animal models that feeding cholesterol-rich, olive oil-based diets causes a hypercholesterolaemic response together with an increase in hepatic cholesterol accumulation (McNamara, 1992). These effects of olive oil were also observed in the present study. In contrast, rapeseed oil, the other MUFA-rich fat studied was as effective as sunflowerseed oil in lowering plasma cholesterol.

The hepatic cholesterol concentration, especially the storage pool of cholesteryl esters was increased with all diets, again mainly the result of excessive dietary cholesterol. The extent of the cholesterol accumulation was affected by the type of dietary fat. Palm stearin resulted in the lowest hepatic total and esterified cholesterol concentrations, even when compared with the other saturated fat-rich diets (butter and coconut oil). Cholesteryl ester accumulation was elevated with olive and sunflowerseed oils and, to a lesser degree, with rapeseed oil. These changes are in keeping with results from previous studies on cholesterol-fed rats, hamsters or guinea-pigs (Jones et al. 1990; Spady et al. 1993; Lin et al. 1994; Jonnalagadda et al. 1995). The present increase in hepatic cholesteryl esters after feeding MUFA- and PUFA-rich diets is consistent with the proposed increase in cholesterol esterification by unsaturated fatty acids, shifting cholesterol from the regulatory pool into the inert storage pool and subsequently restoring the hepatic LDL-receptor activity which generally results in a reduction of plasma LDL-cholesterol (Daumerie et al. 1992; Woollett et al. 1992; Dietschy et al. 1993). The increase in cholesteryl oleate and -linolate in the livers of hamsters fed on sunflowerseed, olive or rapeseed oils evidently reflects the enhancement of acyl-CoA cholesterol acyl transferase (EC 2.3.1.26; ACAT) activity. These particular fatty acids are preferred substrates for this enzyme, which catalyses the esterification of cholesterol to various fatty acids (Spady et al. 1993).

In the present study the hepatic cholesterol accumulation was elevated to the same degree by feeding olive oil or sunflowerseed oil whereas with rapeseed oil the hepatic cholesterol concentration was $14 \%$ lower. Recently, studies using the hamster model revealed a significantly higher accumulation of hepatic cholesterol with olive compared with sunflowerseed or maize oil (Jones et al. 1990; Sessions \& Salter, 1994). These disparate findings in the responses to olive, rapeseed and PUFA-rich oils are most probably the result of different dietary cholesterol intakes. Whereas the previously mentioned studies (Jones et al. 1990; Sessions \& Salter, 1994) used a moderate amount of dietary cholesterol ( 1.0 or $1.2 \mathrm{~g} / \mathrm{kg}$ respectively), an excessive amount of cholesterol $(4 \mathrm{~g} / \mathrm{kg})$ was added to the diets in the present study. There is a differentiating impact of olive oil on hepatic cholesterol accumulation with moderate dietary cholesterol, whereas with extremely high levels of dietary cholesterol the effect of olive oil is dominated by other factors such as the amount of saturated fatty acids. In contrast, rapeseed oil acts more like PUFA-rich oils even when challenged with excessive dietary cholesterol.

Possibly, the different cholesterolaemic effects of olive and rapeseed oils on plasma, and to a lesser extent on hepatic, lipids can be explained by the different fatty acid profiles. Since the amount of 18:2 was adjusted to $2 \%$ en in all diets, one major difference between the olive and rapeseed oil diets was the amount of palmitic acid. Amongst the diets fed in the present study the rapeseed oil diet had the lowest amount of 16:0. Interestingly, the increasing amounts of 16:0 in the diets $(\mathrm{g} / 100 \mathrm{~g}$ total fatty acids: 5.6 with rapeseed oil, 7.6 with sunflowerseed oil, 8.6 with coconut oil, 12.8 with olive oil, 32.6 with butter and 47.5 
with palm stearin) paralleled to a certain extent the increase in plasma cholesterol. It is well established that in the hamster saturated fatty acids, e.g. palmitic acid, in combination with dietary cholesterol cause severe hypercholesterolaemic effects (Spady \& Dietschy, 1988). Recently, another study with cholesterol-fed hamsters also found differences in cholesterol-lowering between oleic acid-rich sunflowerseed oil and olive oil diets, the latter containing almost three times more palmitic acid (Khor \& Tan, 1994).

In addition, rapeseed oil contains a relatively high amount of $\alpha$-linolenic acid (11 $\mathrm{g} / 100 \mathrm{~g}$ total fatty acids) which also could have contributed to cholesterol-lowering. To our knowledge the impact of this particular $n-3$ fatty acid on plasma and hepatic lipids has not been studied in detail in the hamster model. However, $n$-3 PUFA from fish oil caused a substantial increase in plasma cholesterol, especially in VLDL- and LDL-cholesterol in cholesterol-fed hamsters (Cohen et al. 1992; Surette et al. 1992). It seems unlikely, therefore, that $\alpha$-linolenic acid is involved in the distinct cholesterol-lowering by rapeseed oil.

Furthermore, olive and rapeseed oils differ in plant sterol and squalene concentrations. Rapeseed oil contains 2.5 times more plant sterols than olive oil (Jones et al. 1994). Plant sterols may affect cholesterol metabolism indirectly by reducing cholesterol absorption. Squalene may have a direct effect as it is a precursor in cholesterol synthesis. Olive oil contains appreciable amounts of squalene $(1500-9000 \mathrm{mg} / \mathrm{kg}$ oil) which may be partially absorbed and converted to cholesterol (Miettinen, 1993), and thus, mitigate the cholesterollowering action of the MUFA in olive oil.

Finally, daily faecal bile acid excretion was slightly higher with rapeseed compared with olive oil, possibly implying a slightly greater conversion of cholesterol into bile acids. In addition, daily neutral sterol and especially cholesterol excretion in the faeces was also highest in hamsters fed on rapeseed oil, possibly suggesting a reduced cholesterol absorption due to the higher sterol output. These differences in sterol excretion seem insufficient to explain the different responses to rapeseed and olive oils.

In hamsters and possibly in human subjects cholesterol-lowering by olive and rapeseed oils depends largely, it seems, on the amount of dietary cholesterol. Olive oil acts in a cholesterol-lowering manner when dietary cholesterol is low-to-moderate and the LDLreceptors are only partly suppressed as in the studies of Jones et al. (1990) and Sessions \& Salter (1994). In contrast, when the hamster is challenged with an excessive dietary cholesterol load ( $4 \mathrm{~g} / \mathrm{kg}$ as in the present study), and the LDL-receptors are completely shut down, the increase in plasma cholesterol cannot be diminished by feeding olive oil. Probably, restoring the suppressed hepatic LDL-receptor is not only affected by the amount of MUFA but is further influenced by the MUFA : saturated fatty acid ratio. Therefore, rapeseed oil with a more favourable fatty acid profile, because of the lowest amount of saturated fatty acids, has a superior influence on plasma cholesterol compared with olive oil. Rapeseed oil can be considered intermediate between olive and sunflowerseed oils in its fatty acid composition, in the unsaturated : saturated fatty acid ratio and in the plant sterol content. All these together may explain the observed cholesterol-lowering by rapeseed oil, comparable in its magnitude to that of sunflowerseed oil. Likewise, other hamster studies, as well as human studies, have revealed similar cholesterol-lowering effects of rapeseed and maize oils whereas the effect of olive oil on plasma cholesterol was significantly lower (Lichtenstein et al. 1993; Jones et al. 1994, Khor \& Tan, 1994). Taken together, these pieces of evidence would suggest that when cholesterol-rich diets are consumed, rapeseed oil is more effective at lowering plasma lipids than is olive oil, and is comparable in the size of its effects to PUFA. 
Unexpectedly, the incidence of cholesterol gallstones was low in hamsters from all diet groups. Although this phenomenon is not fully understood, it is possible that the hamster strain used in the present study (Charles River lakeview strain from a German breeding facility) was not prone to cholelithiasis. It is well established that the source and genetic background of the animals are important variables when gallstone formation is evaluated (Cohen et al. 1989; Trautwein et al. 1993a). Other factors such as seasonal variation in the susceptibility of gallstone formation could also be involved. Nevertheless, the palm stearin diet, which is rich in 16:0, tended to be more lithogenic than diets rich in other saturated fatty acids (butter and coconut oil). This observation supports previous findings that 16:0rich fats and palmitic acid when added to the diet enhance cholesterol gallstone formation and so have greatest lithogenic effects (Ayyad et al. 1992; Jonnalagadda et al. 1995).

In conclusion, feeding cholesterol-rich diets containing rapeseed and sunflowerseed oils resulted in the lowest plasma cholesterol and triacylglycerol concentrations whereas olive oil failed to demonstrate cholesterol-lowering. The different responses to rapeseed and olive oils after consuming a cholesterol-rich diet are most probably attributable to lower amounts of saturated fatty acids, particularly 16:0, and a higher content of total plant sterols in rapeseed oil. The mechanisms involved remain to be elucidated.

The study was supported in part by grants from UFOP (Union zur Förderung von Öl- und Proteinpflanzen), Bonn, Germany and from 'Stifterfond UNILEVER' des Stifterverbands für die Deutsche Wissenschaft, Essen, Germany.

\section{REFERENCES}

Ausman, L. M., Johnson J. A., Guidry, C. \& Nair, P. P. (1993). Fecal bile acids and neutral sterols in the cottontop tamarin (Saguinus oedipus). Comparative Biochemistry and Physiology 105B, 655-663.

Ayyad, N., Cohen, B. I., Mosbach, E. H. \& Miki, S. (1992). Palmitic acid enhances cholesterol gallstone incidence in Sasco hamsters fed cholesterol enriched diets. Lipids 27, 993-998.

Cohen, B. I., Matoba, N., Mosbach, E. H. \& McSherry, C. K. (1989). Dietary induction of cholesterol gallstones in hamsters from three different sources. Lipids 24, 151-156.

Cohen, B. I., Mosbach, E. H., Ayyad, N., Miki, S. \& McSherry, C. K. (1992). Dietary fat and fatty acids modulate cholesterol cholelithiasis in the hamster. Lipids 27, 526-532.

Dam, H. (1971). Determinants of cholesterol cholelithiasis in man and animals. American Journal of Medicine $51,596-613$.

Daumerie, C. M., Woollett, L. A. \& Dietschy, J. M. (1992). Fatty acids regulate hepatic low density lipoprotein receptor activity through redistribution of intracellular cholesterol pools. Proceedings of the National Academy of Sciences USA 89, 10797-10801.

Dietschy, J. M., Turley, S. D. \& Spady, D. K. (1993). Role of liver in the maintenance of cholesterol and low density lipoprotein homeostasis in different animal species, including humans. Journal of Lipid Research 34, 1637-1659.

Folch, J., Lees, M. \& Sloane Stanley, G. H. (1957). A simple method for the isolation and purification of total lipids from animal tissue. Journal of Biological Chemistry 226, 497-509.

Hayes, K. C., Stephan, Z. F., Pronczuk, A., Lindsey, S. \& Verdon, C. (1989). Lactose protects against estrogeninduced pigment gallstones in hamsters fed nutritionally adequate purified diets. Journal of Nutrition 119 , 1726-1736.

Hayes, K. C., Khosla, P., Kaiser, A., Yeghiazarians, V. \& Pronczuk, A. (1992). Dietary fat and cholesterol modulate the plasma lipoprotein distribution and production of pigment or cholesterol gallstones in hamsters. Journal of Nutrition 122, 374-384.

Heuman, D. M. (1989). Quantitative estimation of the hydrophilic-hydrophobic balance of mixed bile salt solutions. Journal of Lipid Research 30, 719-730.

Jones, P. J. H., Lichtenstein, A. H., Schaefer, E. J. \& Namchuk, G. L. (1994). Effect of dietary fat selection on plasma cholesterol synthesis in older, moderately hypercholesterolemic humans. Arteriosclerosis and Thrombosis 14, 542-548.

Jones, P. J. H., Ridgen, J. E. \& Benson, A. P. (1990). Influence of dietary fatty acid composition on cholesterol synthesis and esterification in hamsters. Lipids 25, 815-820. 
Jonnalagadda, S. S., Trautwein, E. A. \& Hayes, K. C. (1995). Dietary fats rich in saturated fatty acids (12:0), $14: 0$, and 16:0) enhance gallstone formation relative to monounsaturated fat $(18: 1)$ in cholesterol-fed hamsters. Lipids 30, 415-424.

Khor, H. T. \& Tan, D. T. S. (1994). Serum lipid responses to simultaneous alterations in dietary palmitic and oleic acid in the absence and presence of dietary cholesterol in the hamster. Nutrition Research 14, 11911199.

Kim, J. C. \& Chung, T. H. (1984). Direct determination of the free cholesterol and individual cholesteryl esters in serum by HPLC. Korean Journal of Biochemistry 16, 69-77.

Kuroki, S., Cohen, B. I., Carey, M. C. \& Mosbach, E. H. (1986). Rapid computation with the personal computer of the percent cholesterol saturation of bile samples. Journal of Lipid Research 27, 442-446.

Lichtenstein, A. H., Ausman, L. M., Carrasco, W., Jenner, J. L., Gualtieri, L. J., Goldin, B. R., Ordovas, J. M. \& Schaefer E. J. (1993). Effects of canola, corn, and olive oils on fasting and postprandial plasma lipoproteins in humans as part of a National Cholesterol Education Program Step 2 diet. Arteriosclerosis and Thrombosis 13, 1533-1542.

Lin, E. C. K., Fernandez, M. L., Tosca, M. A. \& McNamara, D. J. (1994). Regulation of hepatic LDLmetabolism in the guinea pig by dietary fat and cholesterol. Journal of Lipid Research 35, 446-457.

McNamara, D. J. (1992). Dietary fatty acids, lipoproteins, and cardiovascular disease. Advances in Food and Nutrition Research 36, 253-351.

Miettinen, T. A. (1993). Non-fatty acid components of oils rich in monounsaturated fat. Scandinavian Journal of Nutrition/Näringsforskning 37, 66.

Rossi, S. S., Converse, J. L. \& Hofman, A. F. (1987). High pressure liquid chromatographic analysis of conjugated bile acids in human bile: simultaneous resolution of sulfated and unsulfated lithocholyl amidates and the common conjugated bile acids. Jourmal of Lipid Research 28, 589-595.

Sessions, V. A. \& Salter, A. M. (1994). The effects of different dietary fats and cholesterol on serum lipoprotein concentration in hamsters. Biochimica et Biophysica Acta 1211, 207-214.

Spady, D. K. \& Dietschy, J. M. (1985). Dietary saturated triacylglycerols suppress hepatic low density lipoprotein receptor activity in the hamster. Proceedings of the National Academy of Sciences USA 82, 45264530 .

Spady, D. K. \& Dietschy, J. M. (1988). Interaction of dietary cholesterol and triglycerides in the regulation of hepatic low density lipoprotein transport in the hamster. Journal of Clinical Investigation 81, 300-309.

Spady, D. K., Turley, S. D. \& Dietschy, J. M. (1985). Rates of low density lipoprotein uptake and cholesterol synthesis are regulated independently in the liver. Journal of Lipid Research 26, 465-472.

Spady, D. K., Woollett, L. A. \& Dietschy, J. M. (1993). Regulation of plasma LDL-cholesterol levels by dietary cholesterol and fatty acids. Annual Review of Nutrition 13, 355-381.

Suckling, K. E., Benson, G. M., Bond, B., Gee, A., Glen, A., Haynes, C., \& Jackson, B. (1991). Cholesterol lowering and bile acid excretion in the hamster with cholestyramine treatment. Atherosclerosis 89, $183-190$.

Surette, M. E., Whelan, J., Lu, G. P., Broughton, K. S. \& Konsell, J. E. (1992). Dependence on dietary cholesterol for $n-3$ polyunsaturated fatty acid-induced changes in plasma cholesterol in the Syrian hamster. Journal of Lipid Research 33, 263-271.

Trautwein, E. A., Liang, J. \& Hayes, K. C. (1993a). Cholesterol gallstone induction in hamsters reflects strain differences in plasma lipoproteins and bile acid profiles, Lipids 28, 305-312.

Trautwein, E. A., Siddiqui, A. \& Hayes, K. C. (1993b). Modeling plasma lipoprotein-bile lipid relationships: differential impact of psyllium and cholestyramine in hamsters fed a lithogenic diet. Metabolism 42, 15311540.

Weingard, K. W. \& Daggy, B. P. (1990). Quantification of high-density-lipoprotein cholesterol in plasma from hamsters by differential precipitation. Clinical Chemistry 36, 575.

Woollett, L. A., Spady, D. K. \& Dietschy, J. M. (1992). Saturated and unsaturated fatty acids independently regulate low density lipoprotein receptor activity and production rate. Journal of Lipid Research 33, 77-88. 